\title{
Integrating emotional valence and semantics in the human ventral stream: a hodological account
}

\author{
Sylvie Moritz-Gasser ${ }^{1,2}$, Guillaume Herbet ${ }^{1,2}$ and Hugues Duffau ${ }^{1,2 *}$ \\ Department of Neurosurgery, Gui de Chauliac Hospital, Montpellier University Medical Center, Montpellier, France \\ 2 Team "Plasticity of Central Nervous System, Stem Cells and Glial Tumors," INSERM U1051, Institute for Neuroscience of Montpellier, Saint Eloi Hospital, \\ Montpellier, France \\ *Correspondence: $h$-duffau@chu-montpellier.fr
}

Edited by:

Cornelia Herbert, University Clinic for Psychiatry and Psychotherapy, Germany

Reviewed by:

José Antonio Hinojosa, Universidad Complutense of Madrid, Spain

Keywords: ventral stream, emotion, semantic processing, anatomo-functional connectivity, brain electrostimulation mapping, subcortical pathways

\section{INTRODUCTION}

Accessing the meaning of words to produce and understand language requires the activation of semantic representations. These latter are stored in semantic memory, organizing concepts according to semantic attributes (e.g., the cat mews), and semantic categories (e.g., the cat is an animal). Concerning semantic attributes, non-living concepts (e.g., tools) are processed preferentially according to functional features (e.g., a saw cuts) rather than visual features, whereas living concepts (e.g., animals) are processed preferentially according to visual features (e.g., the cat is little, with sharp ears) rather than functional ones (Warrington and Shallice, 1984). Within these semantic attributes, some are widely shared, independently from our personal history (e.g., the cat mews), and some are linked with our autobiographic memory (e.g., I had a cat when during my childhood). Moreover, most of the concepts have an emotional connotation which, whether it is widely shared (e.g., a black cat brings misfortune) or linked with our personal history (e.g., I love cats because mine was so soft) in a subjective-centered manner, constitutes a semantic attribute, i.e. a defining characteristic (Cato Jackson and Crosson, 2006).

Therefore, not only semantic category and "cold" widely-shared semantic attributes, but also "warm" emotionrelated attributes should be activated to produce or understand a word. Even if the meaning of words may be accessed by a single "cold" semantic processing, words with emotional connotation (widely-shared and/or personal) are more quickly and efficiently processed (Bock and Klinger, 1986), allowing a faster and more accurate lexical access than neutral words (Scott et al., 2009; Méndez-Bértolo et al., 2011; Kissler and Herbert, 2013). It is worth noting that processing wordemotional connotation "differs from the actual experience of emotion: emotional connotation refers to knowledge about the emotional property of an object" (Cato Jackson and Crosson, 2006) and that "emotion modulates word production at several processing stages" (Hinojosa et al., 2010).

Semantic representations forming concepts are more than the simple summation of defining features (Lambon-Ralph et al., 2010). However, how these semantic representations are organized at the neural level is still poorly understood. While some models suggest a distributed organization between a number of interacting cortical associative regions (Turken and Dronkers, 2011), an alternative model proposes an unified organization of semantic representations in an amodal shape in the anterior temporal lobes (ATLs), receiving integrated information from different modality-specific cortical areas. In this latter framework, the ATLs are named "amodal hubs" (Patterson et al., 2007; Lambon-Ralph et al., 2009).

Here, in the light of our clinical observations during picture naming in glioma patients who underwent awake surgery, we bring a new insight on how semantic and personal-emotional information are integrated at the brain systems level, enabling to perform a well-rounded and efficient semantic processing, in order to achieve a complete noetic experience.

\section{A DIRECT AND AN INDIRECT ROUTE FOR SEMANTIC PROCESSING}

We highlighted previously the crucial role of the inferior fronto-occipital fasciculus (IFOF) in semantic processing (Duffau et al., 2013; Moritz-Gasser et al., 2013; Almairac et al., 2014). We proposed this long-association pathway, which comes from the occipital lobe, posterior-lateral temporal areas and parietal cortex, and runs to the orbitofrontal and dorsolateral prefrontal cortices (Catani et al., 2002; Kier et al., 2004; Wakana et al., 2004), as a ventral plurimodal direct route for semantic processing, parallel to an indirect route subserved by the complex inferior longitudinal/uncinate fasciculi (ILF/UF). Indeed, intraoperative mapping during awake surgery for brain glioma (Duffau et al., 2002, 2005) shows that direct electrostimulation of the left IFOF during a naming task always induces semantic disorders (semantic paraphasias or anomias). This semantic disorganization may be either plurimodal (verbal and nonverbal) when stimulating the deep layer of the IFOF, evidenced by the inability for the patient to perform a non-verbal semantic association task, or "only" verbal, when stimulating the superficial layer of the IFOF. Recent studies, based on the Klingler fiber dissection technique, identified two different components of the IFOF: a superficial and dorsal subcomponent, which connects the dorsolateral prefrontal lobe with the superior parietal lobe and the posterior portion of the 
superior and middle occipital gyri; and a deep and ventral subcomponent, which connects the orbitofrontal cortex with the posterior portion of the inferior occipital gyrus and the posterior temporalbasal area (Martino et al., 2010; Sarubbo et al., 2013). This multilayer organization of the IFOF has recently been confirmed by q-ball tractography (Caverzasi et al., 2014). Interestingly, these anatomical descriptions correspond with the cortical network involved in semantic control, namely prefrontal, temporal-basal and parietal areas (Whitney et al., 2011).

Thus, we assumed that the IFOF plays a crucial role in the monitoring of multimodal semantic processing, and we proposed a dynamic dual-stream model of the ventral amodal semantic route, including both the deep and the superficial layers of the IFOF and the indirect (ILF/UF) ventral pathway (Duffau et al., 2013). Based on data issued from intraoperative electrostimulation, we suggested that the IFOF might play a crucial role not only in multimodal semantic processing but beyond, in the awareness of conceptual knowledge, namely noetic consciousness (Moritz-Gasser et al., 2013).

Tractographic studies suggested that semantic processing is underlain by the sole complex ILF/UF (Agosta et al., 2010). The ILF has a vertical component in the parietal lobe, and a horizontal component that lies within the white matter of the occipital and inferior temporal regions (Schmahmann et al., 2007). From the dorso-lateral surface of the occipital lobe, the ILF runs ventro-medially from the posterior lingual and fusiform gyri and dorso-medially from the cuneus. Then the branches run forward to the superior, middle and inferior anterior temporal gyri on the lateral surface, and medially to the amygdala and the parahippocampal gyrus (Catani et al., 2003; Martino and de Lucas, 2014). The ILF seems to be implicated in visual perception, face and object recognition (Catani and Mesulam, 2008a; Fox et al., 2008), reading (Epelbaum et al., 2008) and spoken language (Mummery et al., 1999; Catani and Mesulam, 2008b).

Concerning face/object recognition and reading, it seems that only the posterior part of the ILF ("visual part" corresponding to occipito-inferotemporal fibers) is involved, whereas concerning spoken language (naming), both posterior and anterior parts of the ILF are involved (the former in visual processing of the object or picture, and the latter in "linking object representations to their lexical labels" (Catani and Mesulam, 2008b), by "allowing the semantic system access to stored lexical information" (Foundas et al., 1998; Mummery et al., 1999).

The UF is a ventral associative bundle that connects the ATL and amygdala with the orbitofrontal cortex (Catani et al., 2002; Catani and Thiebaut de Schotten, 2008). It runs inferiorly to the IFOF within the temporal stem, then it splits into a large ventro-lateral branch which terminates in the lateral orbitofrontal cortex and a smaller medial branch which terminates in the frontal pole (Catani et al., 2002; Thiebaut de Schotten et al., 2012). The UF is traditionally considered to be part of the limbic system (Catani et al., 2013; Von Der Heide et al., 2013). Given its connections, functions linked to the UF may concern episodic memory (valuebased updating of stored representations), language (retrieval of proper names for people, some aspects of semantic memory retrieval), and social-emotional processing (valuation of stimuli, emotional meaning of concepts) (Von Der Heide et al., 2013).

We postulate that this indirect pathway (ILF/UF) is involved but not sufficient to perform an efficient semantic processing. We propose that, given their respective cortical terminations, one of the roles of the complex ILF/UF might be to convey critical emotional and mnemonic information associated with words and needed to generate well-rounded supramodal representations of concepts, under the amodal control of the IFOF.

\section{CORTICAL NETWORK AND SUBCORTICAL CONNECTIVITY OF PERSONAL EMOTIONAL-VALUED SEMANTIC PROCESSING DURING LEXICAL ACCESS: PROPOSAL OF A HODOTOPICAL MODEL}

Picture naming requires an early visual processing and recognition by accessing a stored structural description, and then the selection of the corresponding semantic representation or "concept." In parallel with this preverbal processing, appropriate lexical representations or "words" are activated (Ferrand, 1997; Levelt, 2001), thanks to the selection of the most accurate defining features of the semantic representation (Papagno, 2011). Within these defining features or "semantic attributes," some are "cold," widely-shared, and some are "warm," i.e., with an emotional value, itself widely-shared or personal. As mentioned, words with emotional connotation are processed faster and more efficiently than neutral words.

We hypothesize that, if we can access words accurately with only "cold" attributes processing, a well-rounded lexical access will be achieved more efficiently thanks to an integrated processing of words-related emotion. We argue that the indirect ventral semantic stream, subserved by the complex ILF/UF, is the anatomical substrate of this high-level processing, while the direct ventral semantic stream, subserved by the IFOF, is crucial in the monitoring of amodal semantic processing. Thus, we propose an original anatomo-functional model of lexical access, in which all processes (except the early visual processing) are performed in parallel and synchronically.

Visual processing in occipital structures leads to visual recognition thanks to the activation of structural descriptions stored in temporo-basal areas, linked with corresponding semantic representations. During this preverbal stage, information is transmitted via the posterior part of the ILF. Then, to select the appropriate word, corresponding lexical representations are activated following "cold" and "warm" defining features of the semantic representation thanks to a synchronous processing involving the middle temporal gyrus, anterior ventral temporal cortex and temporal pole via the anterior part of the ILFinteracting with orbitofrontal structures via the UF. These parallel processes are supervised and controlled via the IFOF, in an amodal way (Figure 1).

Interestingly, the left ATL seems to be involved in the retrieval of people proper names (Damasio et al., 1996; Papagno and Capitani, 1998; Grabowski et al., 2001). Our model may explain some clinical presentations to the extent that people proper names can only be accessed with their emotional connotation.

Furthermore, it is worth noting that some parts of the distributed cortical network our model highlights have previously 


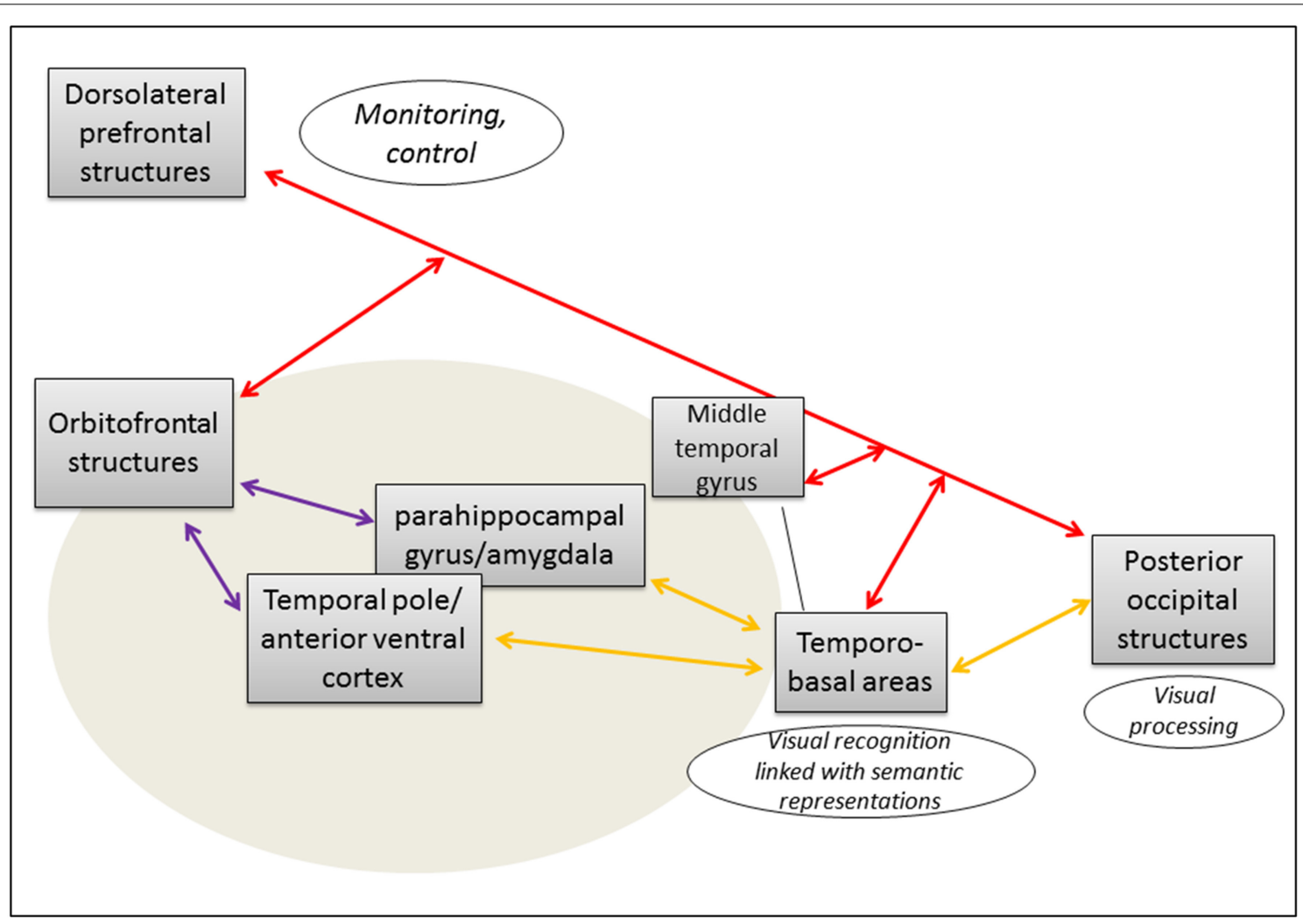

FIGURE 1 | Cortical network and subcortical connectivity of emotional-valued semantic processing during lexical access. Proposal of an anatomo-functional model (the gray oval signalize the network specifically involved in lexical access of emotionally connoted words). Inferior longitudinal fasciculus (ILF); uncinate fasciculus (UF); _ inferior fronto-occipital fasciculus (IFOF). Integration of processes from both the indirect (ILF/UF) and the direct (IFOF) ventral streams is required for an efficient emotional-valued word semantic processing. been proposed as being involved in the processing of word emotional valence in an fMRI study (Kuchinke et al., 2005).

Finally, one of our previous studies based on intraoperative electrostimulation (Mandonnet et al., 2007) suggested that the ILF was not essential in language processing. We proposed that "due to plasticity phenomena induced by slow growing lesion, the function could have been redistributed over the ipsi- or contralateral hemisphere." In other words, the complex ILF/UF is possibly not crucial in semantic processing (because, as mentioned above, an acceptable semantic processing may be performed following only "cold" semantic attributes), and compensable in brain lesions, but this complex is necessary in normal conditions to perform well-rounded, fast and efficient emotional-valued lexicosemantic processing. Nonetheless, the IFOF remains in our model the critical substrate subserving the monitoring and the control of amodal semantic processing. This repeated assumption is in line with the hypothesis of a semantic working memory pathway via the IFOF (Turken and Dronkers, 2011).

In summary, only the integration of synchronous processes from both the indirect and direct ventral streams allows an accurate, efficient and emotionally connoted semantic processing.

\section{CONCLUSION}

We propose an original anatomofunctional model of lexical access, integrating the processing of personal emotional values of words. This model, based on clinical observations of glioma patients undergoing awake surgery and on an extensive review of the literature concerning the anatomo-functional descriptions of white matter associative tracts, puts forward the implication of a large-scale distributed network in this processing. This network might consist of the indirect semantic ventral stream, namely the complex ILF/UF, interconnecting infero-temporo-occipital areas and antero-ventral and medial temporal areas with orbitofrontal structures, which would act synchronically under the amodal monitoring of the direct ventral stream underlain by the IFOF. Integration of processes from both the indirect and direct ventral streams would be required to achieve an emotion-tinged semantic processing, fully and solely human. We may assume that a sole "cold" semantic processing, devoid of any emotional connotation, would entail a disembodied communication, not allowing making sense to situations and to the whole world around us. In other words, a sole "cold" semantic processing wouldn't be a human semantic processing, rich, complex and linked with personal history. We then propose that integration of processes from both the indirect and the direct ventral streams allows a fully achieved, human 
semantic processing leading to a complete noetic experience.

\section{REFERENCES}

Agosta, F., Henry, R. G., Migliaccio, R., Neuhaus, J., Miller, B. L., Dronkers, N. F., et al. (2010). Language networks in semantic dementia. Brain 133, 286-299. doi: 10.1093/brain/awp233

Almairac, F., Herbet, G., Moritz-Gasser, S., de Champfleur, N. M., and Duffau, H. (2014). The left inferior fronto-occipital fasciculus subserves language semantics: a multilevel lesion study. Brain Struct. Funct. doi: 10.1007/s00429-014-07731. [Epub ahead of print].

Catani, M., Howard, R. J., Pajevic, S., and Jones, D. K. (2002).Virtual in vivo interactive dissection of white matter fasciculi in the human brain. Neuroimage 17, 77-94. doi: 10.1006/nimg.2002.1136

Catani, M., Jones, D. K., Donato, R., and Ffytche, D. H. (2003). Occipito-temporal connections in the human brain. Brain 126, 2093-2107. doi: 10.1093/brain/awg203

Catani, M., and Mesulam, M. (2008a). What is a disconnection syndrome? Cortex 44, 911-913. doi: 10.1016/j.cortex.2008.05.001

Catani, M., and Mesulam, M. (2008b). The arcuate fasciculus and the disconnection theme in language and aphasia: history and current state. Cortex 44, 953-961. doi: 10.1016/j.cortex.2008.04.002

Catani, M., and Thiebaut de Schotten, M. (2008). A diffusion tensor imaging tractography atlas for virtual in vivo dissections. Cortex 44, 1105-1132. doi: 10.1016/j.cortex.2008.05.004

Catani, M., Dell'acqua, F., and Thiebaut de Schotten, M. (2013). A revised limbic system model for memory, emotion and behaviour. Neurosci. Biobehav. Rev. 37, 1724-1737. doi: 10.1016/j.neubiorev.2013.07.001

Caverzasi, E., Papinutto, N., Amirbekian, B., Berger, M. S., and Henry, R. G. (2014). Q-ball of inferior fronto-occipital fasciculus and beyond. PLoS ONE 9:e100274. doi: 10.1371/journal.pone.0100274

Cato Jackson, M. A., and Crosson, B. (2006). "Emotional connotation of words: role of emotion in distributed semantic systems," in Progress in Brain Research, Vol. 156, eds S. Anders, G. Ende, M. Junghöfer, J. Kissler, and D. Wildgruber (London: Elsevier), 205-216.

Bock, M., and Klinger, E. (1986). Interaction of emotion and cognition in word recall. Psychol. Res. 48, 99-106. doi: 10.1007/BF00309323

Damasio, H., Grabowsky, T. J., Tranel, D., Hichwa, R. D., and Damasio, A. R. (1996). A neural basis for lexical retrieval. Nature 380, 499-505. doi: $10.1038 / 380499 \mathrm{a} 0$

Duffau, H., Capelle, L., Sichez, N., Denvil, D., Lopes, M., Sichez, J. P., et al. (2002). Intraoperative mapping of the subcortical language pathways using direct stimulations. An anatomo-functional study. Brain 125, 199-214. doi: 10.1093/brain/awf016

Duffau, H., Gatignol, P., Mandonnet, E., Peruzzi, P., Tzourio-Mazoyer, N., and Capelle, L. (2005). New insights into the anatomo-functional connectivity of the semantic system: a study using cortico-subcortical electrostimulations. Brain 128, 797-810. doi: 10.1093/brain/awh423
Duffau, H., Herbet, G., and Moritz-Gasser, S. (2013). Toward a pluri-component, multimodal, and dynamic organization of the ventral semantic stream in humans: lessons from stimulation mapping in awake patients. Front. Syst. Neurosci. 26:44. doi: 10.3389/fnsys.2013.00044

Epelbaum, S., Pinel, P., Gaillard, R., Delmaire, C., Perrin, M., Dupont, S., et al. (2008). Pure alexia as a disconnection syndrome: new diffusion imaging evidence for an old concept. Cortex 44, 962-974. doi: 10.1016/j.cortex.2008.05.003

Ferrand, L. (1997). La dénomination d'objets: théories et données. L'Année Psychologique 97, 113-146. doi: 10.3406/psy.1997.28939

Foundas, A. L., Daniels, S. K., and Vasterling, J. J. (1998). Anomia: case studies with lesion localisation. Neurocase 4, 35-43. doi: 10.1080/13554799808410605

Fox, C. J., Iaria, G., and Barton, J. J. (2008). Disconnection in prosopagnosia and face processing. Cortex 44, 996-1009. doi: 10.1016/j.cortex.2008.04.003

Grabowski, T. J., Damasio, H., Tranel, D., Ponto, L. L., Hichwa, R. D., and Damasio, A. R. (2001). A role for left temporal pole in the retrieval of words for unique entities. Hum. Brain Mapp. 13, 199-212. doi: $10.1002 / \mathrm{hbm} .1033$

Hinojosa, J. A., Méndez-Bértolo, C., Carretié, L., and Pozo, M. A. (2010). Emotion modulates language production during covert picture naming. Neuropsychologia 48, 1725-1734. doi: 10.1016/j.neuropsychologia.2010. 02.020

Kier, E. L., Staib, L. H., Davis, L. M., and Bronen, R. A. (2004). MR imaging of the temporal stem: anatomic dissection tractography of the uncinate fasciculus, inferior occipitofrontal fasciculus, and Meyer's loop of the optic radiation. Am. J. Neuroradiol. 25, 677-691.

Kissler, J., and Herbert, C. (2013). Emotion, Etmnooi, or Emitoon?-Faster lexical access to emotional than to neutral words during reading. Biol. Psychol. 92, 464-479. doi: 10.1016/j.biopsycho.2012. 09.004

Kuchinke, L., Jacobs, A. M., Grubich, C., Vö, M. L., Conrad, M., and Herrmann, M. (2005). Incidental effects of emotional valence in single word processing: an fMRI study. Neuroimage 28, 1022-1032. doi: 10.1016/j.neuroimage.2005. 06.050

Lambon-Ralph, M. A., Pobric, G., and Jefferies, E. (2009). Conceptual knowledge is underpinned by the temporal pole bilaterally: convergent evidence from rTMS. Cereb. Cortex 19, 832-838. doi: 10.1093/cercor/bhn131

Lambon-Ralph, M. A., Sage, K., Jones, R. W., and Mayberry, E. J. (2010). Coherent concepts are computed in the anterior temporal lobes. Proc. Natl. Acad. Sci. U.S.A. 107, 2717-2722. doi: 10.1073/pnas.0907307107

Levelt, W. J. M. (2001). Spoken word production: a theory of lexical access. Proc. Natl. Acad. Sci. U.S.A. 98, 13464-13471. doi: 10.1073/pnas.2314 59498

Mandonnet, E., Nouet, A., Gatignol, P., Capelle, L., and Duffau, H. (2007). Does the left inferior longitudinal fasciculus play a role in language? A brain stimulation study. Brain 130, 623-629. doi: 10.1093/brain/awl361
Martino, J., Brogna, C., Gil-Robles, S., Vergani, F., and Duffau, H. (2010). Anatomic dissection of the inferior fronto-occipital fasciculus revisited in the lights of brain stimulation data. Cortex 46, 691-699. doi: 10.1016/j.cortex.2009.07.015

Martino, J., and de Lucas, E. M. (2014). Subcortical anatomy of the lateral association fascicles of the brain: a review. Clin. Anat. 27, 563-569. doi: $10.1002 / \mathrm{ca} .22321$

Méndez-Bértolo, C., Pozo, M. A., and Hinojosa, J. A. (2011). Word frequency modulates the processing of emotional words: convergent behavioral and electrophysiological data. Neurosci. Lett. 494, 250-254. doi: 10.1016/j.neulet.2011. 03.026

Moritz-Gasser, S., Herbet, G., and Duffau, H. (2013). Mapping the connectivity underlying multimodal (verbal and non-verbal) semantic processing: a brain electrostimulation study. Neuropsychologia 51, 1814-1822. doi: 10.1016/j.neuropsychologia.2013.06.007

Mummery, C. J., Patterson, K., Wise, R. J., Vandenberghe, R., Price, C. J., and Hodges, J. R. (1999). Disrupted temporal lobe connections in semantic dementia. Brain 122 (Pt 1), 61-73. doi: 10.1093/brain/122.1.61

Papagno, C. (2011). Naming and the role of the uncinate fasciculus in language function. Curr. Neurol. Neurosci. 11, 553-559. doi: 10.1007/s11910-0110219-6

Papagno, C., and Capitani, E. (1998). Proper name anomia: a case with sparing of the first letter knowledge. Neuropsychologia 36, 669-679. doi: 10.1016/S0028-3932(97) 00142-5

Patterson, K., Nestor, P. J., and Rogers, T. T. (2007). Where do you know what you know? The representation of semantic knowledge in the human brain. Nat. Rev. Neurosci. 8, 976-987. doi: 10.1038/nrn2277

Sarubbo, S., de Benedictis, A., Maldonado, I. L., Basso, G., and Duffau, H. (2013). Frontal terminations for the inferior frontooccipital fascicle: anatomical dissection, DTI study and functional considerations on a multi-component bundle. Brain Struct. Funct. 218, 21-37. doi: 10.1007/s00429-0110372-3

Schmahmann, J. D., Pandya, D. N., Wang, R., Dai, G. D'Arceuil, H. E., de Crespigny, A. J., et al. (2007). Association fibre pathways of the brain: parallel observations from diffusion spectrum imaging and autoradiography. Brain 130, 630-653. doi: 10.1093/brain/awl359

Scott, G. G., O'Donnell, P. J., Leuthold, H., and Sereno, S. C. (2009). Early emotion word processing: evidence from event-related potentials. Biol. Psychol. 80, 95-104. doi: 10.1016/j.biopsycho.2008.03.010

Thiebaut de Schotten, M., Dell'Acqua, F., Valabregue, R., and Catani, M. (2012). Monkey to human comparative anatomy of the frontal lobe association tracts. Cortex 48, 82-96. doi: 10.1016/j.cortex.2011.10.001

Turken, A. U., and Dronkers, N. F. (2011). The neural architecture of the language comprehension network: converging evidence from lesion and connectivity analyses. Front. Syst. Neurosci. 5, 1-20. doi: 10.3389/fnsys.2011.00001 
Von Der Heide, R. J., Skipper, L. M., Klobuslcky, E., and Oison, I. R. (2013). Dissecting the uncinate fasciculus: disorders, controversies and a hypothesis. Brain 136, 1692-1707. doi: 10.1093/brain/awt094

Wakana, S., Jiang, H., Nagae-Poetscher, L. M., van Zijl, P. C., and Mori, S. (2004). Fiber tract-based atlas of human white matter anatomy. Radiology 230, 77-87. doi: 10.1148/radiol.2301021640

Warrington, E. K., and Shallice, T. (1984). Categoryspecific semantic impairments. Brain 107, 829-853. doi: 10.1093/brain/107.3.829

Whitney, C., Kirk, M., O’Sullivan, J., Lambon Ralph, M. A., and Jefferies, E. (2011). The neural orga- nization of semantic control: TMS evidence for a distributed network in left inferior frontal and posterior middle temporal gyrus. Cereb. Cortex 21, 1066-1075. doi: 10.1093/cercor/bhq180

Conflict of Interest Statement: The authors declare that the research was conducted in the absence of any commercial or financial relationships that could be construed as a potential conflict of interest.

Received: 04 October 2014; accepted: 08 January 2015; published online: 28 January 2015.

Citation: Moritz-Gasser S, Herbet G and Duffau H (2015) Integrating emotional valence and semantics in the human ventral stream: a hodological account. Front. Psychol. 6:32. doi: 10.3389/fpsyg.2015.00032

This article was submitted to Language Sciences, a section of the journal Frontiers in Psychology.

Copyright (c) 2015 Moritz-Gasser, Herbet and Duffau. This is an open-access article distributed under the terms of the Creative Commons Attribution License (CC BY). The use, distribution or reproduction in other forums is permitted, provided the original author(s) or licensor are credited and that the original publication in this journal is cited, in accordance with accepted academic practice. No use, distribution or reproduction is permitted which does not comply with these terms. 